\title{
A Novel way of Basic Probability Assignment Calculation for Multi-sensor Data Fusion
}

\author{
Younghwan Oh \\ Department of Information and Communication, Korea Nazarene University \\ \{yhoh@kornu.ac.kr\}
}

\begin{abstract}
The Basic Probability Assignment is also called mass function. The BPA should basically include the uncertainty and obscurity of the context of the real world to recognize and reduce the uncertainty in the result of the arithmetic operation. The BPA makes a decision to make it possible the arithmetic operation between different heterogeneous signals or data. This study proposed calculation of the BPA by getting a clue from a policy that recognizes at a certain time interval and reports changes worth reporting. Usually, 'the size of changes' is often the base in a decision of meaningful recognition of events reported to the host. Like this, what aspect the value sensed with the lapse of time has as compared to that sensed in previous time is related to the context. Thus, in deciding the BPA, reflecting this point is relevant. This study analyzed the change of the proposed sensing value to decide the BPA, and based on this, it proved that contexts could be inferred.
\end{abstract}

Keywords: Context Inference, Basic Probability Assignment

\section{Introduction}

Lots of sensors and diverse networks are necessary for the Internet of things. Multiple sensors have been developed for functions and performances. The networks used in the Internet of things are divided broadly into mobile network and wireless data communication network. Sensors in the Internet of things attached or distributed in all existing objects in the real world obtain and report information about the status of objects or peripheral environment. One of the purposes of the Internet of things consisting of these sensors and communication networks is the inference of contexts. The context inference is the assumption of the objects aimed by the sensors or change to focus, occurring or progressing in the periphery based on the sensors' sensing signals and data on a reasonable basis. The context inference cannot but be accompanied by ambiguity and uncertainty. The context inference requires immediacy. It is significant to use multiple sensor data fusion in order to remove or reduce its ambiguity and uncertainty. Multiple sensor data fusion methods are divided broadly into four: First, a method based on probability theory; second, one using a Kalman filter; third, one using an artificial neural network; and fourth, one using the fuzzy theory. Among the four methods, the method using the Kalman filter has been used for the purpose of predicting the track of movement of mobile vehicles or air routes of flying objects. If the other three kinds of methods can be used as a data fusion method necessary for the inference of contexts, among them, what plays a fundamental role in the method based on the probability theory and fuzzy theory is to calculate and decide the basic probability assignment function or mass function. It is very significant to decide the basic probability assignment function for the multiple sensor data fusion that contributes greatly to the performance improvement of the context inference $[9,10]$. 


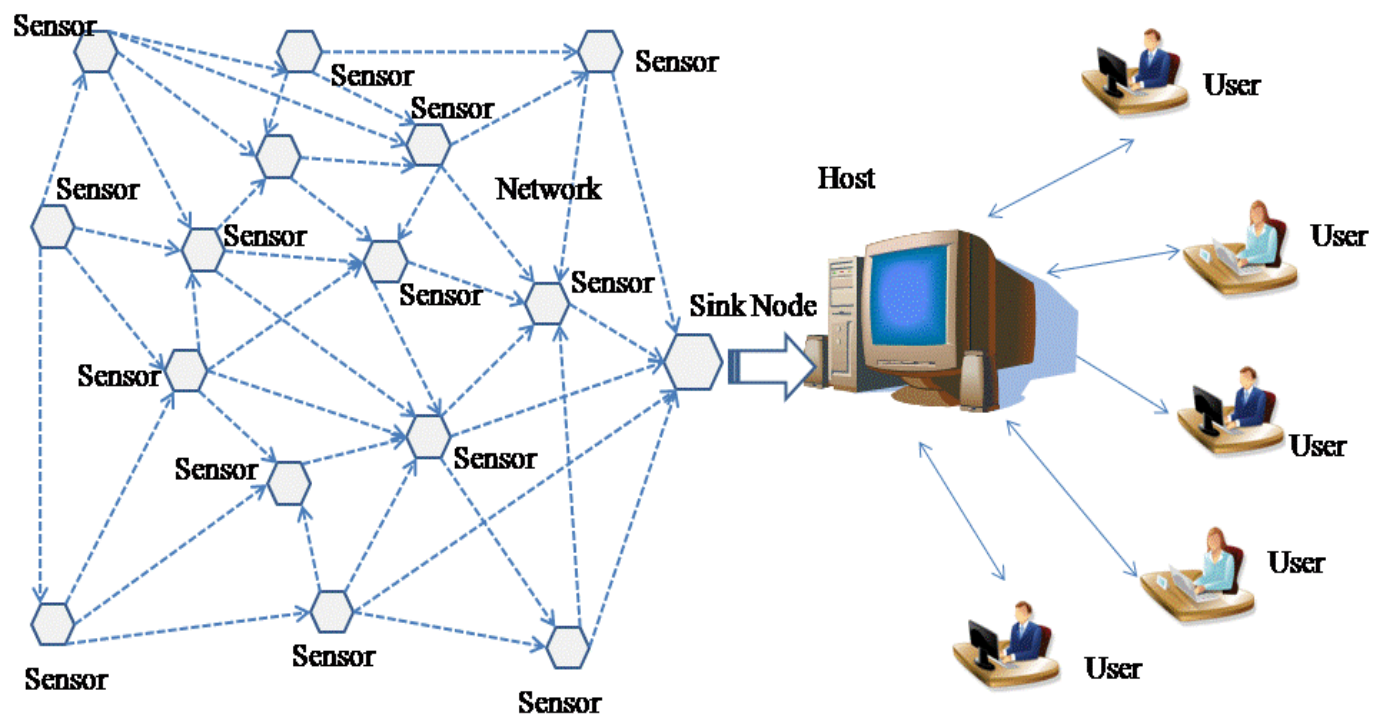

Figure 1. Wireless Sensor Network

This study proposes a method to decide the basic probability assignment function that contributes greatly to the context inference on the Internet of things. The method of deciding the basic probability assignment function in this study is based on the environment in which there are uncertainty and obscurity, and it should reduce this uncertainty. Apart from numerically receiving sensing and reporting data on piecemeal sensors, it should closely identify the relationship between the aspect of the changes of the measured sensor values and the actual context, and based on this, it should decide the basic probability assignment function. This is because it should execute data fusion that has great contribution to the context inference. The basic probability assignment function fundamentally makes a decision to make it possible the arithmetic operation between different heterogeneous signals or data. It targets the objects that are physically inconsistent, thus, they cannot be compared and integrated, or their correlation degree cannot be calculated. This study got a clue from the data filtering policy that recognizes at a certain time interval, and then reports changes worth reporting. A sensor mote does not report all values sensed to the host in order to reduce its power consumption. Usually, power is consumed mostly doing communication activity. In a decision of meaningful recognition of events reported to the host, 'the size of changes' is often the base. Like this, what aspect the value sensed with the lapse of time has as compared to that sensed in previous time is related to the context. Thus, in deciding the basic probability assignment function, reflecting this point is relevant.

This study is composed of the following: Chapter 2 summarized related research; Chapter 3 proposed a method of deciding the basic probability assignment function of this study. It is experimented and verified based on the theory proposed in Chapter 4, and Chapter 5 draws conclusions. 


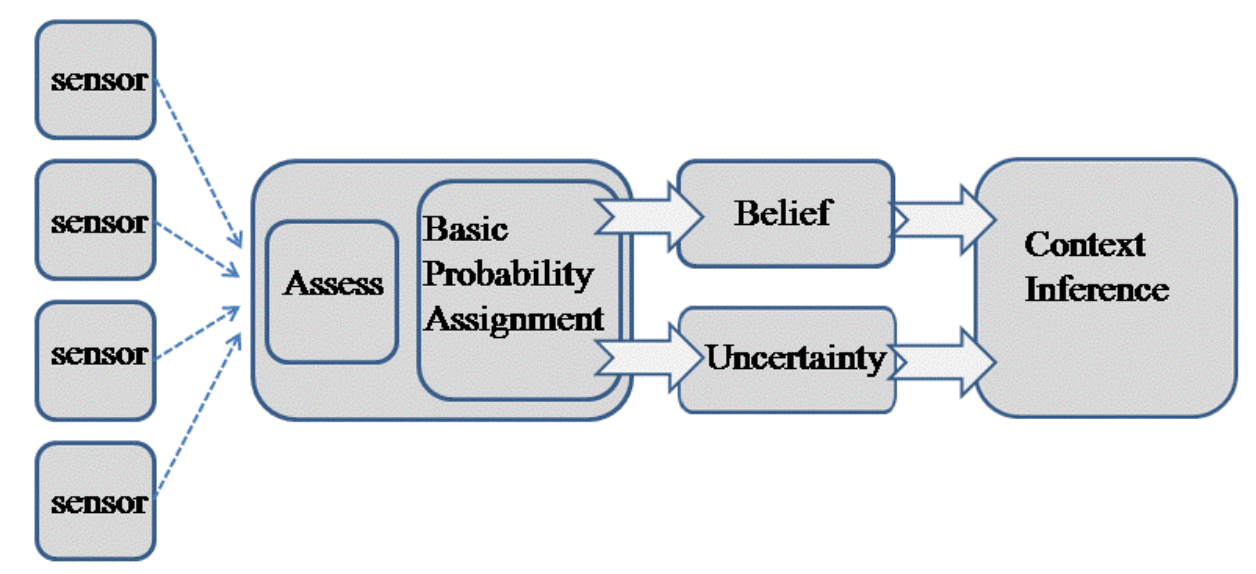

Figure 2. Basic Probability Assignment and Context Inference

\section{Related Research}

Determining basic probability assignment is significant in the multi-sensor data fusion by using Fussy Theory or Dempster-Shafer Theory. The multi-sensor data fusion processing with DST is that the belief value and plausibility value of each focal element calculated from BPA of each focal element is determined, and context of real world can be infer from distinguish the belief value and uncertainty value of each focal element after getting uncertainty value of the difference between belief value and plausibility.

\subsection{Requirements of DST}

Suppose that in a DST, the whole set consisting of mutually exclusive propositions is $\Theta$ and that $2^{\theta}$ is the power set of $\Theta$, which includes all possible propositions, the basic probability assignment $m$ assigned to an interest element has four kinds of the following characteristics $[3,4]$.

1) $\mathrm{m}: 2^{\theta} \rightarrow[0,1]$

2) $m(\varnothing)=0$

3) $0 \leq m(A) \leq 1$

4) $\sum_{\mathrm{A} \subseteq \theta} \mathrm{m}(\mathrm{A})=1$

Using the basic probability assignment, individual propositions, that is, the value of quantified total reliability allotted to the combination of the elements of interest, $A$ and the probability value of the elements of interest can be found, which are the basis of the context inference $[1,2]$.

\subsection{Methods of deciding a BPA}

So many ways of studies on determining BPA have been conducted.

T. Ali et al., proposed a method of deciding a BPA in the context in which minimal value, maximum value and arithmetic operator in an approximate value are recognized [3].

W. Jiang proposed a method of calculating a BPA by measuring the properties of the objects to analyze and the distance between the tested sample data [4].

Z. Zuo et al., proposed a method of calculating a BPA using a rough set theory based on the random set theory and BP neural network [5], and A. O. Boudraa et al., 
proposed a method of calculating a BPA using an image segment method using fuzzy membership level pixel found in an image histogram [6].

Suh, Donghyeok et al., granted weighted value by sensor in multiple sensor data fusion in a wireless sensor network based on Dempster-Shafer's evidence theory for the life safety guarantee of the disabled and compared whether the performance of the context inference improved.

They could infer improved context information as a weighted value was given to the information to fusion when a method of guaranteeing the life safety of the disabled through the multiple sensor data fusion technique [7,8].

\section{Determining Basic Probability Assignment for Efficient Data Fusion}

A process of collecting information using various diverse sensors to recognize or infer contexts using sensors and drawing highly accurate information and knowledge by fusion of it is necessary. The data fusion has a different characteristic from data combination that removes common sensing information of various sensors for a specific event or context. Of the data fusion techniques, DST and fuzzy theory may have an advantage in inferring diverse contexts as compared to the data fusion using a Kalman filter and may be advantageous in terms of processing speed as compared to the artificial neural network. DST and fuzzy theory is very dependent on the basic probability assignment function. The basic probability assignment function is also called mass function, and decisions of good or bad results through data fusion using DST and fuzzy theory are made depending on how to decide the basic probability assignment function.

The basic probability assignment function should basically include the uncertainty and obscurity of the context of the real world to recognize and reduce the uncertainty in the result of the arithmetic operation. The relationship between the aspects of the changes of the sensor values sensed, measured and reported by sensors and the actual context should be closely identified, and decisions of the basic probability assignment function should be made based on this. This is because the Internet of things or a ubiquitous sensor network ultimately aims to recognize contexts and provide intelligent and personalized follow-up services. Therefore, the basic probability assignment function decision should allow good execution of data fusion with high contribution to inferring contexts.

The basic probability assignment function fundamentally makes a decision to make it possible the arithmetic operation of different heterogeneous signals or data. The values sensed and reported by the heterogeneous sensors are totally different from those sensed and reported by physically different types of sensors. Thus, it is difficult to compare and integrate them and difficult to analyze their correlation degree. The basic probability assignment function aims to calculate these heterogeneous sensor values together, which are incalculable. In constructing a network, generally, there is middleware between hardware and software. Middleware in a sensor network is divided into in-network side middleware and server-side middleware, and in the in-network side middleware, a sensor mote can set up a time interval sensing a peripheral context and has a criterion for the decision of whether to transmit the sensed value to the host server and a policy on filtering the requirements for reporting of the sensed values. This study gets a clue from the data filtering policy that recognizes at certain time interval and reports changes worth reporting. A sensor mote does not report all values sensed to the host, and this is to reduce its power consumption. Usually, 'the size of changes' is often the base in a decision of meaningful recognition of events reported to the host. Like this, what aspect the value sensed with the lapse of time has as compared to that sensed in previous time is related to the context. Thus, in deciding the basic probability assignment function, reflecting this point is relevant.

The following procedures show the principles and procedures of deciding the basic probability assignment function proposed in this study in a formula. 
$D S=<D_{1}, D_{2}, \cdots, D_{t}>$ : $t$ is current time and $D_{t}$, data, which is expressed as follows:

$$
D_{t}=\left(s_{t}, i_{t}, u_{t}\right)
$$

Where $S_{t}$ is the sound value at $t ; I_{t}$, the infrared light sensor value at $t$; and $U_{t}$, the ultrasonic wave sensor value at $t$.

$\Delta_{s t}=s_{t}-s_{t-1}, \Delta_{i t}=i_{t}-i_{t-1}, \Delta_{u t}=u_{t}-u_{t-1}$

When the amount of changes is defined as above,

if $\alpha$ is a regularizing function, the regularizing functions of each sensor value can be calculated as follows:

$$
\alpha_{s t}=\sigma\left(\delta_{s t}, \Delta_{s t}, \overline{s_{t}}\right), \alpha_{i t}=\sigma\left(\delta_{i t}, \Delta_{i t}, \overline{\bar{l}_{t}}\right), \alpha_{u t}=\sigma\left(\delta_{u t}, \Delta_{u t}, \overline{u_{t}}\right)
$$

The following function is taken by the transformation of a sigmoid function.

$$
\sigma(x, y, z)=1 /\left(x e^{-y / z}+1\right)
$$

Each mean is defined as follows:

$\overline{s_{t}}=1 /(t-1) \sum_{k=1}^{t-1} s_{k}, \overline{l_{t}}=1 /(\mathrm{t}-1) \sum_{k=1}^{t-1} i_{k}, \overline{u_{t}}=1 /(t-1) \sum_{k=1}^{t-1} u_{k}$

The variant values are defined as follows:

$$
\begin{aligned}
& \delta_{s t}=1 /(t-1) \sum_{k=1}^{t-1}\left(\overline{\mathrm{s}_{\mathrm{t}}}-s_{k}\right)^{2}, \delta_{i t}=1 /(\mathrm{t}-1) \sum_{k=1}^{t-1}\left(\overline{\mathrm{l}_{\mathrm{t}}}-i_{k}\right)^{2}, \\
& \delta_{u t}=1 /(t-1) \sum_{k=1}^{t-1}\left(\overline{\mathrm{u}_{\mathrm{t}}}-u_{k}\right)^{2}
\end{aligned}
$$

Now, to decide a BPA, Function m: $2^{\mathrm{D}_{t}} \rightarrow[0,1]$ is defined as follows:

$$
m(A)=\left(\sum_{k \in A} \alpha_{k}\right) / \alpha_{k}
$$

This algorithm bears in mind the multi-sensor data fusion for the values reported to the host through a filtering policy on the values sensed after three types of sensors sense the targets of sensing at a certain time interval. 


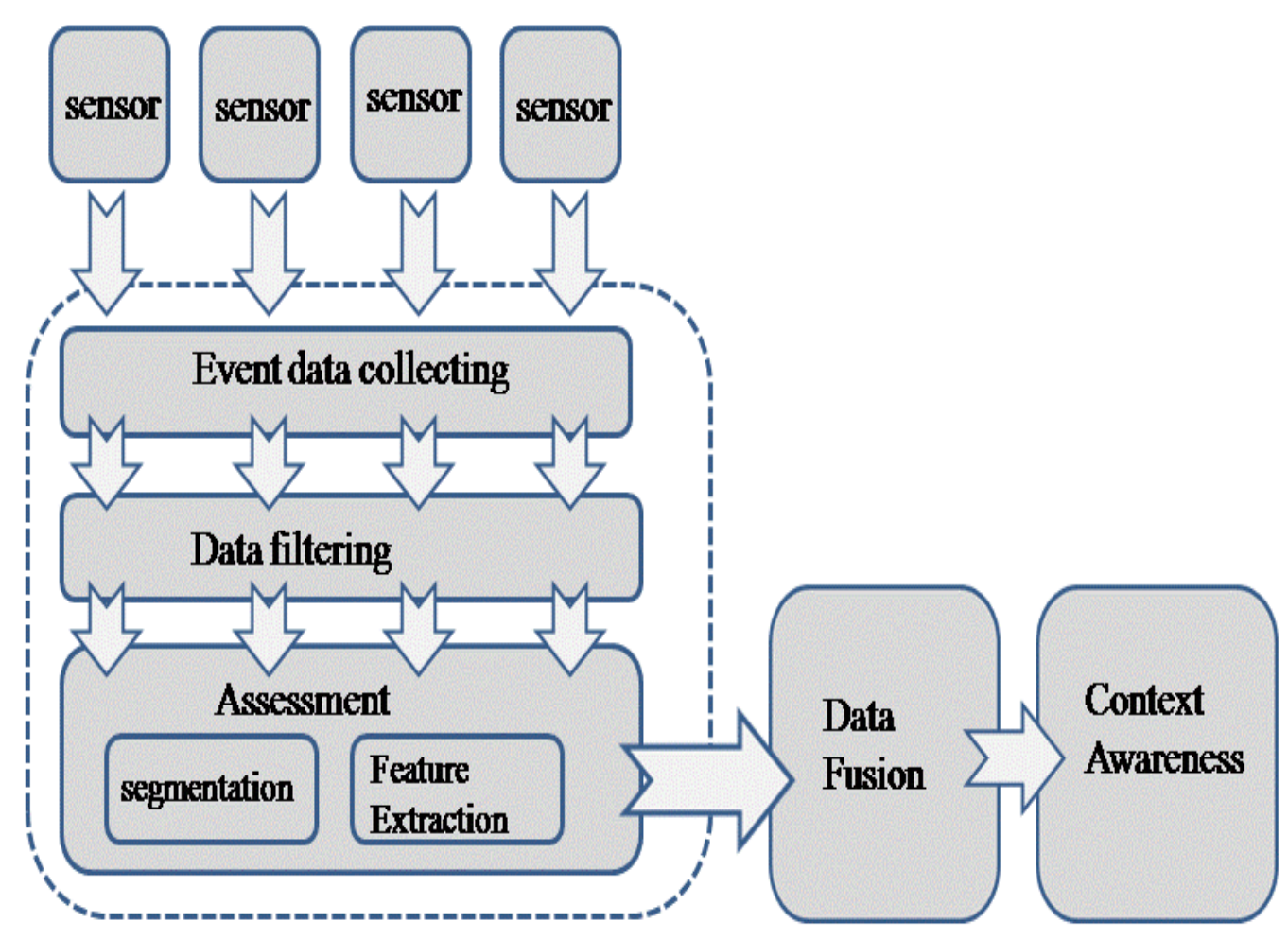

Figure 3. Determining Basic Probability Assignment for Context Awareness

\section{An Experiment and Evaluation}

The details of the experiment on the algorithm proposed in Chapter 3 are as follows: Three kinds of sensors, sound, ultrasonic wave and infrared light sensors were employed, and the time interval at which each sensor senses was set at 0.1 second, and when each sensor consistently reported the values measured to the host, they were not infinitely stored, but cut at a certain time interval, and the basic probability assignment function was calculated and decided at the relevant time.

\section{Experiment procedure}

- The Sensor network is consisted with the sound sensor, ultrasonic wave sensor and the infrared sensor.

- Sensing event data are obtained each 0.1 seconds.

- The BPA with event data and the belief and the uncertainty with the DST are calculated.

- The contexts are inferred by analyzing the belief and the uncertainty of each focal element.

The following Table 1 shows the data values obtained in the host by sensors' measurement and reporting. 
Table 1. The Sensed Data from the Three Sensor Motes

\begin{tabular}{cccc}
\hline time & distance & sound & Lux \\
\hline 1 & 200 & 0 & 1003 \\
2 & 192 & 20 & 1002 \\
3 & 200 & 0 & 998 \\
4 & 196 & 0 & 995 \\
5 & 199 & 0 & 988 \\
6 & 192 & 0 & 978 \\
7 & 194 & 0 & 967 \\
8 & 199 & 0 & 960 \\
9 & 200 & 0 & 952 \\
10 & 196 & 5 & 945 \\
\hline
\end{tabular}

The following Table 2 shows the results basic probability assignment with the data of Table 1.

Table 2. The Basic Probability Assignments of each Focal Element

\begin{tabular}{llllllll}
\hline time & $\mathrm{h} 1$ & $\mathrm{~h} 2$ & $\mathrm{~h} 3$ & $\mathrm{~h} 1+\mathrm{h} 2$ & $\mathrm{~h} 1+\mathrm{h} 3$ & $\mathrm{~h} 2+\mathrm{h} 3$ & $\mathrm{~h} 1+\mathrm{h} 2+\mathrm{h} 3$ \\
\hline $0 \sim 10$ & 0.000026 & 0.621014 & 0.000047 & 0.154172 & 0.000018 & 0.155911 & 0.068813 \\
$10 \sim 20$ & 0.000504 & 0.624244 & 0.000247 & 0.153165 & 0.000352 & 0.154178 & 0.067309 \\
$20 \sim 30$ & 0.000067 & 0.621035 & 0 & 0.154781 & 0.000018 & 0.155292 & 0.068807 \\
$30 \sim 40$ & 0.000018 & 0.620738 & 0.000003 & 0.155058 & 0.000009 & 0.155234 & 0.068938 \\
$40 \sim 50$ & 0.000032 & 0.621334 & 0.000019 & 0.154721 & 0.000024 & 0.155172 & 0.068698 \\
$50 \sim 60$ & 0.000069 & 0.619251 & 0.000024 & 0.155982 & 0.000042 & 0.155149 & 0.069483 \\
$60 \sim 70$ & 0.000049 & 0.621 & 0.000042 & 0.154928 & 0.000042 & 0.155129 & 0.068812 \\
$70 \sim 80$ & 0.00001 & 0.619376 & 0.000008 & 0.155675 & 0.000008 & 0.155459 & 0.069464 \\
$80 \sim 90$ & 0.000025 & 0.619928 & 0.000009 & 0.155796 & 0.000015 & 0.154981 & 0.069245 \\
$90 \sim 100$ & 0.000058 & 0.621018 & 0.000054 & 0.155092 & 0.000055 & 0.154928 & 0.068796 \\
\hline
\end{tabular}

The basic probability assignment should basically involve the uncertainty and belief of the context of the real world to recognize and make less the uncertainty in the result of the calculation. The basic probability assignment determination plays a important role in allowing better processing of multi-sensor data fusion that contributes to the context awareness and inference.

The basic probability assignment basically makes a decision to make it possible the calculation between heterogeneous signals or data from sensor motes. The event values sensed and reported by various sensors have different properties. It is difficult to integrate them and difficult to analyze their correlation and contribution degree. This experiment showed the way of calculation of the basic probability assignment by getting a clue recognizes at a certain time interval and reports changes worth reporting. A sensor mote does not report all values to the host through the network, and this is to reduce its electrical power consumption. Commonly, 'the size of changes' is often the base of meaningful recognition of events information reported to the host. Like this, what aspect the value sensed with the lapse of time has as compared to that sensed in previous time is related to the context. Thus, in determining the basic probability assignment, reflecting this point is relevant. 


\section{Conclusion}

The basic probability assignment function is also called mass function, and decisions of good or bad results through data fusion using DST and fuzzy theory are made depending on how to decide the basic probability assignment function.

The basic probability assignment function should basically include the uncertainty and obscurity of the context of the real world to recognize and reduce the uncertainty in the result of the arithmetic operation. The basic probability assignment function decision plays a crucial role in allowing good execution of data fusion that greatly contributes to the context inference.

The basic probability assignment function fundamentally makes a decision to make it possible the arithmetic operation between different heterogeneous signals or data. The values sensed and reported by heterogeneous various sensors have physically different properties. It is difficult to compare and integrate them and difficult to analyze their correlation degree. This study proposed calculation of the basic probability assignment function by getting a clue from a policy that recognizes at a certain time interval and reports changes worth reporting. A sensor mote does not report all values sensed to the host, and this is to reduce its power consumption. Usually, 'the size of changes' is often the base in a decision of meaningful recognition of events reported to the host. Like this, what aspect the value sensed with the lapse of time has as compared to that sensed in previous time is related to the context. Thus, in deciding the basic probability assignment function, reflecting this point is relevant.

This study analyzed the change of the proposed sensing value to decide the basic probability assignment function, and based on this, it proved that contexts could be inferred.

\section{Acknowledgements}

This research was supported by the Korea Nazarene University Research Grants 2014.

\section{References}

[1] T. Gu, H. Pung and D. Zhang, "A Service-Oriented Middleware for Building Context -Aware Services", Journal of Network and Computer Applications, vol. 28, no. 1, (2005) January, pp. 1-18.

[2] S. Vijayarani and P. Jothi, "An Efficient Clustering Algorithm for Outlier Detection in Data Streams", International Journal of Advanced Research in Computer and Communication Engineering, vol. 2, no. 9, (2013) September.

[3] T. Ali and P. Dutta, "Methods to Obtain Basic Probability Assignment in Evidence Theory", International Journal of Computer Applications, vol. 38, no. 4, (2012), pp. 46-51.

[4] W. Jiang, "A New Method to Determine BPA in Evidence Theory", Journal of Computers, vol. 6, no. 6, (2011)

[5] Z. Zuo,Y. Xu, and G. Chen, "A New Method of Obtaining BPA and Application to the Bearing Fault Diagnosis of Wind Turbine", Proceedings of the 2009 International Symposium on Information Processing(ISIP'09), (2009) August, pp. 368-371.

[6] A. O. Boudraa, A. Bentabet, F. Salzenstein and L. Guillon, "Dempster-Shafer's Basic Probability Assignment Based on Fuzzy Membership Functions", Electronic Letters on Computer Vision and Image Analysis, vol. 4, no. 1, (2004).

[7] D. H. Suh and S. S. Yoon, "Weighting Method Based on Event Frequency for Multi-sensor Data Fusion in Wireless Sensor Network for the People with Disability", Journal of Assistive Technology, vol. 5, no. 1, (2011), pp. 37-47.

[8] D. H. Suh and S. S. Yoon, "Multi-sensor Data Fusion for the Indoor Living Security of Children with a Disability", Journal of Assistive Technology, vol. 4, no. 2, (2010), pp. 35-56.

[9] V. N. Hamed, Z. Kamran and N. Nasser, "Context-Aware Middleware Achitecture for Smart Home Environment”, International Journal of Smart Home, vol. 7, no. 1, (2013), pp. 77-86.

[10] W. Mo and Z. Ding, "A Novel Template Weighted Match Degree Algorithm for Optical Character Recognition”, International Journal of Smart Home, vol. 7, no. 3, (2013) May, pp. 261-270.

[11] P. Thi Bach Hue, S. Wohlgemuth, I. Echizen and Nguyen, "An Experimental Evaluation for a New Column - Level Access Control Mechanism for Electronic Health Record Systems", International Journal of $\mathrm{u}$ - and e - Service, Science and Technology, vol. 4, no. 4, (2011) December, pp. 1-14. 
[12] Y. Gu, L. Sun and J. Wang, "Comparative Analysis of Single and Mixed Spatial Interpolation Methods for Variability Prediction of Temperature Prediction", International Journal of Hybrid Information Technology, vol. 6, no. 1, (2013) January, pp. 67-76.

\section{Author}

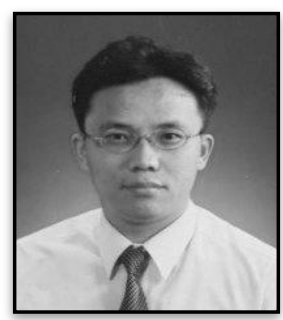

YoungHwan Oh, he received the M.S. degrees in computer science from Inha University in 1997 and the Ph.D. in computer science from Inha University in 2001. His research interests included in stream data processing and data fusion in wireless sensor network. He is a professor in Korea Nazarene University from 2002.

His research interests are included in data fusion in wireless sensor network and data mining. 
International Journal of Control and Automation Vol. 7, No. 11 (2014) 